\title{
Regulatory Futures in Retrospect
}

To appear in: Johan Eriksson, Michael Gilek and Christina Rudén (eds.) Regulating Chemical Risks: European and Global Challenges, Springer, 2009.

\section{Authors and affiliations:}

Willem Halffman, w.halffman@gmail.com

Science, Technology, and Policy Studies (STəPS), Twente University

Roland Bal, r.bal@bmg.eur.nl

Department of Health Policy and Management, Erasmus University Medical Centre

\section{Author bio:}

Willem Halffman lectures policy sciences at the department of Science, Technology, Health and Policy Studies (STEHPS) at Twente University. He is a co-ordinator of the PhD training programme of the Netherlands Graduate Research School of Science, Technology, and Modern Culture.

Roland Bal is a professor of Healthcare Governance at the Department of Health Policy and Management of the Erasmus University Medical Centre.

\section{Address for correspondence:}

Willem Halffman, w.halffman@gmail.com

Science, Technology, and Policy Studies (STəPS), Twente University

PO Box 217, 7500 AE Enschede, The Netherlands

Tel +31 (0)534893221 / 3353, Fax +31 (0)534892159 
3948 words (excluding abstract and references)

\begin{abstract}
In our 1998 volume 'The Politics of Chemical Risk: Scenarios for a regulatory Future' we envisioned four ideal typical scenarios for the future of European chemicals policies. The scenarios focused on the nature of expertise (seen either as a universal or a localised phenomenon) and the organisation of the boundary between science and policy (as either diverging or converging). The four scenarios were titled International Experts, European Risk Consultation, European Coordination of Assessment, and Europe as a Translator. For all four scenarios, we hypothesized internal dynamics and articulated dilemmas related to the development of the sciences contributing to chemical assessment, the relation between the EU and member states and the role of the public.

In this contribution, we look back on our four scenarios fifteen years later, to see which ones have materialized and to explore whether the dilemmas we saw have indeed surfaced. We conclude that the International Experts scenario by and large has materialized and explore some of the underlying tensions and dynamics in this development.
\end{abstract}




\section{Regulatory futures}

In the winter of 1995 we gathered thirty experts in an Amsterdam hotel to get to the bottom of the fundamental misunderstandings over chemical risk regulation. Half of the experts were natural scientists working on chemical risk issues: toxicologists, regulatory risk assessors, experts from regulatory agencies. The other half were social scientists who had been studying regulatory decision making, trying to analyse the frames and assumptions underlying regulation, and in some cases even challenging those assumptions, academically or in activism.

We were young and our plan was ambitious. Both of us were doing $\mathrm{PhD}$ research on the organisation of the science/policy boundary in chemical licensing and standard setting, either in occupational health (Bal, 1999), or environmental hazards of chemicals (Halffman, 2003). We imagined that, in the absence of concrete economic stakes or disagreement over specific substances, we could at least have a meaningful debate about the paradigm of risk assessment, the boundary between science and policy in regulatory regimes, and the world views underlying them. We had also hoped to consider some alternative ideas to organise regulatory regimes. To focus the debate, we aimed at the construction of four scenarios for the future organisation of regulatory chemical hazard assessment. The scenarios focused on the nature of expertise (seen either as universal or imbued with local context, such a national regulatory traditions) and the organisation of the boundary between science and policy (as either sharp and diverging or flexible and converging). The results were published in a book, which included both the presentations and an account of the debates at the event (Bal \& Halffman, 1998).

We now write almost a decade and a half later. New issues have appeared on the regulatory agenda since: toxicity of nano-materials, the unravelling of BSE, chemical persistence, or endocrine disruption. Meanwhile also, the organisation of regulatory expertise has witnessed some important shifts, such as with the introduction of the REACH scheme and the creation of the European Chemicals Agency, as extensively documented in this book. When the invitation came from the editors of this volume to look back and 
reflect on how far the regulatory debate has come since, we were keen to make use of the occasion, although our research interests had moved on since.

In this chapter, we will give an account of the issues at stake around regulatory expertise, as we encountered them in our Amsterdam discussions and how we saw possible futures at the time. Second, we want to compare our expectations to regulatory developments since, as reflected in this volume. Last, we will once again try to look forward and explore regulatory futures once again.

\section{The future, as it was}

At the Amsterdam workshop, we presented four scenarios, entitled International Experts, European Risk Consultation, European Coordination of Assessment, and Europe as a Translator. For all four scenarios, we hypothesized internal dynamics and articulated dilemmas related to the development of the sciences contributing to chemical assessment, the relation between the EU and member states and the role of the public (Bal, 1998; Halffman \& Bal, 1998).

The scenarios were based on what we saw as important tensions in regulatory regimes. First, we saw a tension between universalising risk assessment and a growing attention for local conditions of regulation. Risk assessment was clearly moving towards harmonisation, in Europe, but also beyond, as stated emphatically by the representatives of international initiatives. Regulatory experts insisted that they were making progress with shared standards for how to assess chemical hazards, so that companies would not have to repeat permit applications with slightly different testing requirements.

At the same time, there were indications of a counter-tendency, pointing out the need for political choice in chemical hazard assessment, particularly with respect to the definition of what needs to be protected (e.g. all biodiversity of a stream or merely interesting species and their ecological support). As the political assumptions at the basis of what seems technical risk assessments were being made explicit, it seemed like they would require rich political institutions to address. Similarly, different economic conditions could be conducive to a continued importance of national governments, leaving at best an intergovernmental logic for limited regulatory cooperation. 
The second tension we saw, was between those who defended a clear-cut demarcation of the work of experts and regulatory policy makers on the one hand, and those who questioned the wisdom of this insistence. This divide ran roughly along the split between natural scientists and social scientists, but the two did not coincide completely.

The argument for a strict maintenance of a science/policy boundary was complex. The regulatory scientists generally insisted on the importance of universalist principles of science and on standardised methodologies as guarantees for regulatory independence. Essentially, they argued, as many still do, that experts should be kept apart from politics to be able to do their job, taking understandable and admirable professional pride in their work. With such a separation, a rational process of risk assessment would be possible, while undesired intervention of superstition, emotion, political passion, or private interest could be kept at bay.

The counter-argument form the social scientists (and science and technology studies in particular) were also varied. One was that private interest cannot be kept at bay by well intentioned principles, but that industry (to call it by its name) always manages to be present on the science-side of the divide more than other interests. Another argument was that the insistence on a strict separation based on standardised methods leads to the exclusion of un-standardised knowledge of adverse effects. (At the time endocrine disruption was becoming a major issue that was not covered in standard methods). In addition, a strategy to insist on a strict boundary would always face a challenge of hypocrisy, as risk assessment is unable to purify itself completely from all politics, all value-laden assumptions. Rather than to see a universal scientific method, social scientists pointed to the co-construction of methods by both scientists and regulatory agencies together, in fact at the very construction of entire fields of scientific expertise in the shadow of policy agencies such as the US Environmental Protection Agency or the OECD.

The answer of regulatory experts to such issues is that they can show that nothing untoward is going on by being transparent about their work. Rather than to hide behind secretive deliberations that relied on personalised expertise, regulatory experts were begin to learn to document their evaluations. However, at the same time, governments 
noticed that publishing an overwhelming amount of technical information can just as much create inaccessibility to regulatory assessments as a total lack of public information. An increasing transparency creates its own intransparancies. In addition, transparency only works if the premises of regulatory action are shared by all involved, which was exactly the problem. To use the example of endocrine disruption again: no amount of transparency could solve the problem that critics wanted this included as a relevant endpoint for testing, while regulatory experts could only point at the lack of standard methodology to assess such effects. Similarly, critics pointed out the chemical-bychemical assessment, ignoring the accumulated effect of chemicals already in circulation - a fundamental problem that continues to pose a serious challenge to all chemical regulation that considers exposure rates in assessments.

As an alternative, we proposed to look at the relation between experts and nonexperts more symmetrically, for example by arguing that the principles by which experts (dis)trust each other are really not that different from the principles by which lay people (dis)trust experts. For example, experts rely on the reputation of other experts to assess whether a claim requires additional questioning, are more critical of a discrepant finding, or a finding that leads to higher costs, just as lay people do. With such arguments, we argued that more dialogical relations between processes of expertise and of value consideration were at least worth considering.

In the scenario International Experts, we extrapolated the development of standards harmonised on a European level, organised around a strict boundary between science and politics. We saw this to imply the creation of a European chemical risk assessment agency, effectively internationalising expertise, fixing lingering differences in style and approach between EU countries. This is the scenario that most resembled the development of EU chemical regulation since. Even though, with hindsight, this may seem like a necessary development, at the time it was not. It seemed at least as likely that European chemical assessment would remain under national control, the European Coordination of Assessment scenario, so that countries maintained national resources to perform trusted assessments. Harmonization would then have gradually proceeded 
through increased co-ordination of procedures, further protocolization, and increased expert cooperation.

Our alternatives that suggested a more flexible attitude about the science/policy boundary seem further away from actual developments. In "Europe as a translator" we saw a regulatory role for Europe as a mediator between chemical regulation and debate as they were differently framed in various countries. In the last scenario, European risk consultation, expertise shifts to a European level, but provisions are made for controversy through consultation with national experts. Assessment is routinised where possible, but evaluation details are publicly available, allowing for a shift of assessment to more consultative procedures.

On paper, it seems as if the "International Experts" scenario has become the reality. However, the tensions we identified in this scenario remain present. The key element of trying to maintain a strict boundary between risk assessment and risk management is that reason is mobilised to tame the beast of politics. The assumption is that we can define the rules of the reason game beforehand (test standards, assessment protocols, expert decision rules) and then hold all parties involved to these rules once the game is played. However, no game is played like that. Players contest the interpretation of the rules, the application of the rule to a particular case, whether a particular case is subject to the rules, will try to question the rules, change them, and - if all else fails - undermine them, sabotage implementation, or just refuse to play. For most players, the foundational rule that risk assessment must be separated from risk management can become just as questionable as the other rules. Even though chemical regulation driven by expert risk assessment may 'fix' risk regulation in principle, the question remains whether they also do in practice.

\section{New key tensions in chemical regulations: controversy}

The key test for REACH is not whether it will be able to assess thousands of chemicals. The real question is whether it will withstand escalated conflict over a handful of lucrative or strategically important chemicals. Risk assessment procedures in the past have been notoriously bad at anticipating and accommodating contestation. When we brought toxicologists and social scientists together at our meeting, the fiercest debates 
(and the most fundamental misunderstandings) were about the framing of risk research problems, rather than excessive influence or malpractice in regulatory assessments.

Let us pay some extra attention to this point, for it is an extremely important one. Base regulatory contestation accuses regulators of inappropriate sympathies, intentional bias, or even outright corruption. The large majority of regulatory experts are of good faith and would equally condemn such practices. Most contestation of regulatory expertise is not of this base level. Rather, citizens ask questions such as: Are you sure that your findings also hold for children and pregnant women? Have you actually measured that in the soil, or is this a model? What will happen to this substance if it should escape from your laboratory (even if you claim it never will)? Such questions ask whether the risk assessments have been appropriately framed, whether an assessment protocol (by necessity a simplified representation of the world) covers all the relevant processes in the world, who has decided which processes should be considered relevant, and what kind of certainty is appropriate for action to be taken. The GMO debate offers a nice example: for the opponents the question is not just what GMOs will do in the soil or the human bowls, but also what they will do to the power relations between farmers and seed producers, an issue well beyond the expertise of your hard-working, honest, and sincere toxicologist.

Chemical risk assessment is a means to contain such conflicts. It defines a series of relevant endpoints and exposure processes in the world that we will accept as relevant, while others (such as changing property relations) are not. The boundary between risk assessment and risk regulation is not just a boundary that keeps dirty politics out of disinterested science, but also a boundary that prevents new concerns from making the assessment process unpredictable, for applicants, policy makers, as well as environmentalists. Such new concerns can be new health or environmental concerns, but also new arguments for mitigating factors or alleged over-estimation of risk. The alternatives to this strict boundary are basically propositions that try to find conditions for a reasonable debate about what should be considered relevant consequences. Chemical controversies are not just about whether the facts are right, but more interestingly also about whether these are the right facts. 
The question for REACH is whether it will be able to deal with these framing issues. In the next section we discuss some underlying tensions that question the ability of the REACH framework to do so in a sustainable way.

\section{New key tensions in chemical regulations: complexity}

Looking at the development of chemical regulation with the benefit of distance, some key developments can be identified. Most striking is the growing complexity of the regulatory regime. $\mathrm{REACH}$ involves more tests, testing more endpoints, and has more complex decision rules. For example, the extension of environmental effects from acute and chronic effects to persistence as a cause for concern shows how regulatory assessment is trying to include more of the complexity around chemicals interacting with the world.

However, this complexity is not just the result of a regulatory system that tries to mirror the complexity of the world out there. From a more political perspective, it also reflects the complexity of the negotiations around chemical regulation. Environmental concerns have insisted on new endpoints in the micro-politics of technical meetings and grass-root campaigning, such as with endocrine disruptors. Industrial organisations have argued for flexibility, regulatory restraint, or even de-regulation, such as with low volume chemicals. Different EU member states have argued the case for 'their' industries. The complexity of the regulatory regime therefore also shows the complexity of the compromises that seemed necessary to prevent conflict escalation.

At the base of this approach lies a particular strategy for dealing with chemical hazards. This strategy is utilitarian at heart, as has been argued poignantly by philosopher and activist Anne Chapman (Chapman, 2007; Halffman, 2009, forthcoming). The utilitarian logic states that the free enterprise of actors will lead to a collective good, provided that unwanted consequences ('externalities') are contained, typically through rules. In other words: the basic premise for chemical regulatory regimes is that chemical producers are free to pursue their commercial activity, provided these activities to not cause unacceptable harm, as far as specified in general rules that are policed, ultimately, by the state. The rules have to be general, as they have to create a 'level playing field' for all players, that is: equal competitive conditions in an international chemical market. The question of which harms are unacceptable and should be caught in general rules, has 
dominated the EU debate over chemical regulation, for example on the issue of whether mere persistence (rather than toxicity) constitutes a harm.

The growing complexity of regulatory regimes is a problem in itself. First, the complexity of regulation can create a distorted playing field, where only companies capable of maintaining a large staff of regulatory specialists can participate. Rather than to create a free market, complexity can become a means to corner the market and avoid competition. For these reasons, the largest players in a market with complex regulation may even support increasing regulation, as it allows them to take control. The objection is a recurrent one, as smaller firms are concerned about the ability to compete, or small innovators, such as in the ecological pesticides sector, fear they will not be able to pass the regulatory post. Regulations that claim to create a level playing field can thus become self-defeating.

Growing complexity also implies a threat of growing regulatory cost, especially as complex regulation may make it hard to assess regulatory overheads beforehand. The objection that has recently also been raised against REACH, not only in terms of financial cost, but also test animal lives (Hartung \& Rovida, 2009), but is one that has been made since the birth of regulatory regimes, usually from the side of industry. In the markets for pesticides, but also pharmaceuticals, it is often claimed that high regulatory costs stifle innovation or reduce profitability. From a public perspective also, growing complexity raises collective costs, through the need to maintain regulatory agencies, monitoring, and enforcement.

To a certain extent, such objections have been met: with exceptions for small volume chemicals to allow innovation, public regulatory costs being reclaimed through registration fees, with support for innovation through public research funds, or with attempts to harmonise chemical regulation internationally, such as through the creation of a European regulatory agency, rather than to rely on many national ones. However, such solutions have also created additional complexities, exacerbating rather than solving some of these problems.

The growing complexity of chemical regulation also constitutes a problem for the democratic order. The claim that the fairness of regulatory policies is guaranteed by a public, transparent process becomes more and more unreal as this process itself is so 
complex that only a small group of experts can really understand the intricacies of the regulatory filigree. Even fifteen years ago, experts in regulatory agencies were already worried that they were outmatched by the intricate knowledge of regulatory procedure of industry experts. Citizens are therefore increasingly dependent on third parties and their experts in order to assure them that just decisions are being made. At the same time, inexplicable regulatory issues become less and less appealing to the public spaces where citizens discuss the collective good: how many journalists would dare tackle a regulatory decision under REACH for the evening news?

Regulatory complexity also reduces the possibility for effective policy intervention. Complex rules create new loopholes, new possibilities for delay, exception, exemption, objection, appeal. In this respect, chemical regulatory policy has an appalling track record. Effective regulatory action on a chemical of major significance typically takes several decades between early warning and effective policy action, see DDT, PCBs, dioxins, asbestos, phtalates, or anti-fouling paints. It is quite disconcerting that it does not seem to matter much whether a refined regulatory apparatus is in place or not. Most major cases eventually require ad-hoc policy intervention anyway, with specific compromises and measures, specific regulations, and large amounts of non-standard research into unanticipated chemical effects through unforeseen pathways. A coldhearted look at the history of chemical regulation has to ask why such a complex regulatory apparatus has lead to so few effective regulatory actions, especially with respect to chemicals already in use.

Lastly, complexity itself can become a source of hazards, as Charles Perrow has showed for complex technological systems (Perrow, 1999). Where complexity undermines overview and an ability to see how rules affect the concrete life of the regulated subject, regulation can become its own worst enemy, creating a false sense of security. There are also political risks in complexity. Contradicting rules may undermine effective regulatory action, while the resulting absurd regulatory effects are easy pickings for populist anti-regulatory rhetoric that may delegitimize risk policy as a whole. (Health and safety regulations are notorious for unintended contradictions in regulatory requirements; e.g. the floor that has to be both smooth for hygiene and rough so as not to be slippery). In the US, the populist use of regulatory costs and unintended regulatory 
consequences have contributed to undermine effective regulatory innovation for the last decade (e.g. the unfavourable comparison of the US Toxic Substance Control Act and the REACH programme in chapter @ @). Thus, as one example of the possible political risk of complex regulations, regulatory complexity risks creating the conditions for a regulatory backlash.

Regulatory complexity is likely to increase further. One of the major gaping holes in chemical risk assessment is substance interaction. We still largely assess hazards of chemicals one by one, ignoring the fact that, for example, toxic chemicals are added to a world where a lot of toxic chemicals circulate already. We thereby ignore additive effects, let alone mutually reinforcing effects. There are a few exceptions, such as greenhouse gases, such as carbon dioxide, methane, or nitrous oxide. We have learnt to express greenhouse gas effects in terms of their warming effect. That is: we assess risk from the perspective of the receptor, in this case the atmosphere. Environmentalists have long argued that something similar should be done for chemicals and concerns have been raised about cumulative neurotoxic effects of organophosphates. Experts have already recommended to look at toxic equivalents in food (Committee on toxicity of chemicals in food, 2002; Gezondheidsraad, 2002; Health Council of the Netherlands, 2004). Similarly, in the past, we have had to group organochlorine pesticides because of similar effects, doxins, or man-made fibres. Even if we were to identify a handful of key receptors in the human body, a handful in the physical environment, and a handful in the biological environment, the re-assessment of all chemicals from the perspective of these receptors would present an almost impossible task.

Complexity in chemical regulation is also likely to increase because the forces that drive it are still there. Experts continue to research new pathways, continue to develop new tests, or further refine exposure models. The main purpose of maintaining a competitive internal chemical market requires general rules, rather than case-by-case judgement. Every compromise over assessment procedures is embedded in new protocols. Furthermore, societal, including industry interests, are bound to further drive the complexity of regulation as each new controversy tends to add new rules to existing ones. 
It would be wrong to see our argument as a pro-industry plea for deregulation. First, because regulatory simplification is not necessarily pro-industry. In fact, the most radical de-regulation proposal on chemicals was made in circles of Greenpeace, suggesting that perhaps we have enough chemicals already and we should consider a ban on new chemicals. Second, de-regulation would be meaningless as long as there is no significant alternative to deal with the hazards created by the growing human circulation of chemicals through the world, man-made or not.

The growing complexity of regulation may be presented as an increasing correspondence between the complexity of the world and the complexity of assessment protocols, but we would argue that there is a limit to this development.

\section{Conclusions}

In this reflection on the developments of and in chemicals regulation in Europe since we wrote our scenarios more than 10 years ago, we have argued that the course of the developments has by and large followed the International Experts scenario that we then sketched. The separation between risk assessment and risk management has hardened in this period and the regulation of chemicals has increasingly become an international, European, rather than a local affair. We have also argued that some of the key tensions that we then saw resulting from this scenario are still present. Framing issues remain contentious and the increasing complexity of regulation proves to be transparent only for a few experts, thus threatening the democratic character of chemicals regulation. Whether this will result in a backlash in the future remains to be seen, but the conditions for this are most certainly present. 


\section{References}

Bal, R. (1998). Boundary Dynamics in Dutch Standard Setting for Occupational Chemicals. In R. Bal \& W. Halffman (Eds.), The politics of chemical risk: scenarios for a regulatory future (pp. 75-103). Dordrecht: Kluwer Academic Publishers.

Bal, R. (1999). Grenzenwerk: Over het organiseren van normstelling voor de arbeidsplek (Vol. 6). Twente: Twente University Press.

Bal, R., \& Halffman, W. (Eds.). (1998). The Politics of Chemical Risk: Scenarios for a Regulatory Future. Dordrecht: Kluwer.

Chapman, A. (2007). Democratizing Technology: Risk, Responsibility \& the Regulation of Chemicals. London: Earthscan.

Committee on toxicity of chemicals in food, c. p. a. t. e. (2002). Risk assessment of mixtures of pesticides and similar substances. London: Food Standards Agencyo. Document Number)

Gezondheidsraad. (2002). Blootstelling aan combinaties van stoffen. The Hague: Health Council of the Netherlandso. Document Number)

Halffman, W. (2003). Boundaries of Regulatory Science: Eco/toxicology and aquatic hazards of chemicals in the US, England, and the Netherlands, 1970-1995. Unpublished doctoral thesis, University of Amsterdam, Amsterdam.

Halffman, W. (2009, forthcoming). Republican Chemicals (Review of Anne Chapman, Democratizing Technology, London: Earthscan 2007). Journal of Risk Research.

Halffman, W., \& Bal, R. (1998). The politics of chemical risk: Scenarios for regulatory policy. In R. Bal \& W. Halffman (Eds.), The Politics of Chemical Risk (pp. 303322). Dordrecht: Kluwer Academic Publishers.

Hartung, T., \& Rovida, C. (2009). Chemical regulators have overreached. Nature, 460(27 August), 1080-1081.

Health Council of the Netherlands. (2004). Pesticides in food: assessing the risk to children. The Hague: Health Council of the Netherlandso. Document Number)

Perrow, C. (1999). Normal Accidents: living with high-risk technologies. Princeton: Princeton University Press. 